\title{
Topologically protected quantum states and quantum computing in Josephson junctions arrays
}

\author{
L.B. loffe \\ Center for Materials Theory, Department of Physics and Astronomy, Rutgers University \\ 136 Frelinghuysen Rd., Piscataway NJ 08854, USA \\ Landau Institute for Theoretical Physics, 2 Kosygina Str., Moscow 117940, Russia \\ M.V. Feigel'man \\ Landau Institute for Theoretical Physics, 2 Kosygina Str., Moscow 117940, Russia \\ E-mail: feigel@landau.ac.ru \\ B. Douçot \\ Laboratoire de Physique Théorique et Hautes' Energies, CNRS UMR 7589, \\ Universités Paris 6 et 7, 4 Place Jussieu, Paris 75252 Cedex 05, France
}

Received January 5, 2004

\begin{abstract}
We review recent results on a new class of Josephson arrays which have non-trivial topology and exhibit a novel quantum states at low temperatures. One of these states is characterized by long range order in a two Cooper pair condensate and by a discrete topological order parameter. The second state is insulating and can be considered as a result of evolution of the former state due to Bose-condensation of usual superconductive vortices with a flux quantum $\Phi_{0}$. Quantum phase transition between these two states is controlled by variation of external magnetic field. Both the superconductive and insulating states are characterized by the presence of $2^{K}$-degenerate ground states, with $K$ being the number of topologically different cycles existing in the plane of the array. This degeneracy is «protected» from the external perturbations (and noise) by the topological order parameter and spectral gap. We show that in ideal conditions the low order effect of the external perturbations on this degeneracy is exactly zero and that deviations from ideality lead to only exponentially small effects of perturbations. We argue that this system provides a physical implementation of an ideal quantum computer with a built in error correction. A number of relatively simple «echo-like» experiments possible on small-size arrays are discussed.
\end{abstract}

PACS: 74.50.+r, 85.25.-j

\section{Introduction}

Quantum computing [1,2] is in principle a very powerful technique for solving classic «hard» problems such as factorizing large numbers [3] or sorting large lists [4]. The remarkable discovery of quantum error correction algorithms [5] shows that there is no problem of principle involved in building a functioning quantum computer. However, implementation still seems dauntingly difficult: the essential ingredient of a quantum computer is a quantum system with $2^{K}$ (with $K \gg 100$ ) quantum states which are degenerate (or nearly so) in the absence of external pertur- bations and are insensitive to the «random» fluctuations which exist in every real system, but which may be manipulated by controlled external fields with errors less than $10^{-6}$. Moreover, the standard schemes of error-corrections (assuming error rate of order $10^{-6}$ ) require very big system sizes, $K \sim 10^{4}-10^{6}$, to correct the errors (i.e., the total number of all qubits is by factor of 100-1000 exceeds the number of qubits needed to perform computational algorithm in the «ideal» conditions of no errors). If frequency of errors could be reduced by orderes of magnitude, the conditions for residual errow-corrections would becomes 
much less stringent, and the total size of the system, $K$, would be much smaller [6].

Insensitivity to random fluctuations means that any coupling to the external environment neither induces transitions among these $2^{K}$ states nor changes the phase of one state with respect another. Mathematically, this means that one requires a system whose Hilbert space contains a $2^{K}$-dimensional subspace (called «the protected subspace» [7-9]) within which any local operator $\hat{O}$ has (to a high accuracy) only state-independent diagonal matrix elements:

$$
\langle n|\hat{O}| m\rangle=O_{0} \delta_{m n}+o(\exp (-L)),
$$

where $L$ is a parameter such as the system size that can be made as large as desired. It has been very difficult to design a system which meets these criteria. Many physical systems (for example, spin glasses [10]) exhibit exponentially many distinct states so that the off-diagonal matrix elements of all physical operators between these states are exponentially small. In such systems the longitudinal relaxation of a superposition of these states is very slow. The absence of the transverse relaxation which is due to the different diagonal matrix elements $O_{m m}-O_{n n}$ is a different matter: it is highly non-trivial requirement that is not satisfied by usual physical systems (such as spin glasses) and which puts systems satisfying it in a completely new class.

One very attractive possibility, proposed in an important paper by Kitaev [7] and developed further in [11] involves a protected subspace [8,9] created by a topological degeneracy of the ground state. Typically such degeneracy happens if the system has a conservation law such as the conservation of the parity of the number of «particles» along some long contour, and the absence of any local order parameter. Physically, it is clear that two states that differ only by the parity of some big number that cannot be obtained from any local measurement are very similar to each other. Cartoon example of this idea can be presented as follows. Consider two locally flat surfaces, one with a topology of simple cylinder, whereas another one is the Moebius stripe, and imagine an observer moving on one of these surfaces. Clearly, the only way to decide on which surface the observer is located is to walk the whole loop around the stripe and find himself at the same point (then the surface is a cylinder) or on the other side of the surface (if it is Moebius stripe).

The model proposed in [7] has been shown to exhibit many properties of the ideal quantum computer; however before now no robust and practical implementation was known. In a recent paper we and others proposed a Josephson junction network which is an implementation of a similar model with protected de- generacy and which is possible (although difficult) to build in the laboratory [12].

In this paper we review our recent results on further development of ideas of Ref. 12. We propose a new Josephson junction network that has a number of practical advantages (shorter account of this approach can be found in $[13,14])$. This network operates in a phase regime (i.e., when Josephson energy is larger than the charging energy), which reduces undesired effects of parasitic stray charges. All Josephson junctions in this array are similar which should simplify the fabrication process. This system has $2^{K}$-degenerate ground states «protected» to even higher extent than in [12]: matrix elements of local operators scale as $\varepsilon^{L}$, where $\varepsilon \leq 0.1$ is a measure of non-ideality of the system's fabrication (e.g., the spread of critical currents of different Josephson junctions and geomertical areas of different elementary cells in the network). The new array does not require a fine tuning of its parameters into a narrow region. The relevant degrees of freedom of this new array are described by the model analogous to the one proposed in Ref. 7.

In physical terms, the array we propose may exist in two different phases: i) topological superconductor, that is a superfluid of $4 e$ composite objects, and ii) topological insulator that is a superfluid of superconductive vortices with a flux quantum $\Phi_{0}=h c / 2 e$. The topological degeneracy of the ground state i) arises because $2 e$ excitations have a gap. Indeed, in such system with the geometry of an annulus, one extra Cooper pair injected at the inner boundary can never escape it; on the other hand, it is clear that two states differing by the parity of the number of Cooper pairs at the boundary are practically indistinguishable by a local measurement. In the ground state ii) the lowest excitation is a «half-vortex» (i.e., vortex with a flux $\left.\Phi_{0} / 2\right)$, and topological double-degeneracy appears due to the possibility to put a half-vortex inside the opening, without paying any energy.

Below we first describe the physical array, with the «topological superconductor» state, identify its relevant low energy degrees of freedom and the mathematical model which describes their dynamics. We then show how the protected states appear in this model, derive the parameters of the model and identify various corrections appearing in a real physical system and their effects. Then we discuss generalization of this array that is needed to obtain in a controllable way a second phase of «topologically insulator». Finally, we discuss how one can manipulate quantum states in a putative quantum computer based upon those arrays, and the physical properties expected in a small arrays of this type. 
We remark that the properties of the excitations and topological order parameter exhibited by the system we propose here are in many respects similar to the properties of the ring exchange and frustrated magnets models discussed recently in [8,9,15-25].

\section{Array 1: Topological superconductor}

The basic building block of the lattice is a rhombus made of four Josephson junctions with each side of the rhombus containing one Josephson contact, these rhombi form a hexagonal lattice as shown in Fig. 1. We denote the centers of the hexagons by letters $a, b \ldots$ and the individual rhombi by $(a b),(c d) \ldots$, because each rhombus is one-to-one correspondence with the link $(a b)$ between the sites of the triangular lattice dual to the hexagonal lattice. The lattice is placed in a uniform magnetic field so that the flux through each rhombus is $\Phi_{0} / 2$. The geometry is chosen in such a way that the flux, $\Phi_{s}$ through each David's star is a half-integer multiple of $\Phi_{0}: \Phi_{s}=$ $=\left(n_{s}+1 / 2\right) \Phi_{0}{ }^{*}$. Finally, globally the lattice contains a number, $K$, of big openings (the size of the opening is much larger than the lattice constant, a lattice with $K=1$ is shown in Fig. $1, a$ ). The dimension of the protected space will be shown to be equal $2^{K}$. The system is characterized by the Josephson energy, $E_{J}=(\hbar / 2 e) I_{c}$, of each contact and by the capacitance matrix of the islands (vertices of the lattice). We shall assume (as is usually the case) that the capacitance matrix is dominated by the capacitances of individual junctions, we write the charging energy as $E_{C}=e^{2} / 2 C$. The «phase regime» of the network mentioned above implies that $E_{J}>E_{C}$. The whole system is described by the Lagrangian

$$
\mathcal{L}=\sum_{(i j)} \frac{1}{16 E_{C}}\left(\dot{\varphi}_{i}-\dot{\varphi}_{j}\right)^{2}+E_{J} \cos \left(\varphi_{i}-\varphi_{j}-a_{i j}\right),
$$

where $\varphi_{i}$ are the phases of individual islands and $a_{i j}$ are chosen to produce the correct magnetic fluxes. The Lagrangian (1) contains only gauge invariant phase differences, $\varphi_{i j}=\varphi_{i}-\varphi_{j}-a_{i j}$, so it will be convenient sometimes to treat them as independent variables satisfying the constraint $\Sigma_{\Gamma} \varphi_{i j}=$ $=2 \pi \Phi_{\Gamma} / \Phi_{0}+2 \pi n$, where the sum is taken over closed loop $\Gamma$ and $n$ is arbitrary integer.

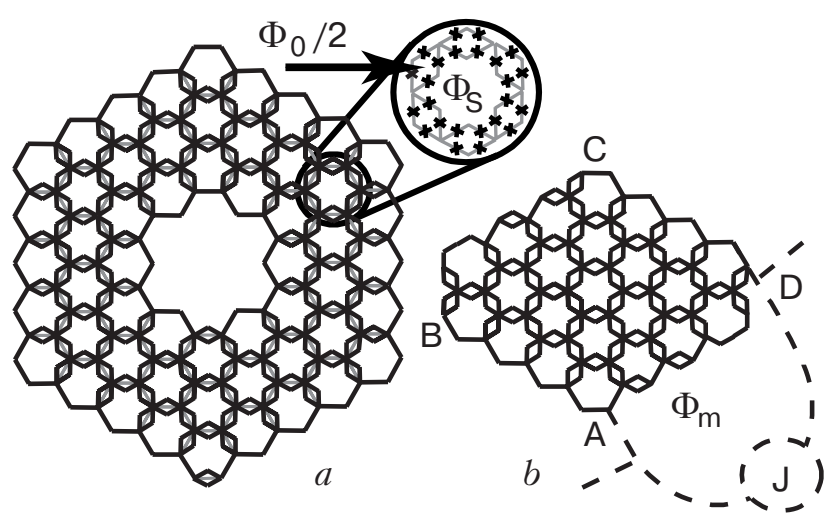

Fig. 1. Examples of the proposed Josephson junction array. Thick lines show superconductive wires, each wire contains one Josephson junction as shown in detailed view of one hexagon. The width of each rhombi is such that the ratio of the area of David's star to the area of one rhombi is odd integer. The array is put in magnetic field such that the flux through each elementary rhombus and through each David's star (inscribed in each hexagon) is half integer. Thin lines show the effective bonds formed by the elementary rhombi. The Josephson coupling provided by these bonds is $\pi$-periodic. Array with one opening, generally the effective number of qubits, $K$ is equal to the number of openings. The choice of boundary condition shown here makes superconducting phase unique along the entire length of the outer (inner) boundary, the state of the entire boundary is described by a single degree of freedom. The topological order parameter controls the phase difference between inner and outer boundaries. Each boundary includes one rhombus to allow experiments with flux penetration; magnetic flux through the opening is assumed to be $(1 / 2+m) \Phi_{0} / 2$ with any integer $m(a)$. With this choice of boundary circuits the phase is unique only inside the sectors $A B$ and $C D$ of the boundary; the topological degree of freedom controls the difference between the phases of these boundaries. This allows a simpler setup of the experimental test for the signatures of the ground state described in the text, e.g., by a SQUID interference experiment sketched here that involves a measuring loop with flux $\Phi_{m}$ and a very weak junction $J$ balancing the array (b).

As will become clear below, it is crucial that the degrees of freedom at the boundary have dynamics identical to those in the bulk. To ensure this one needs to add additional superconducting wires and Josephson junctions at the boundary. There are a few ways to do this, two examples are shown in Fig. 1, $a$ and Fig. 1, $b$ : type I boundary (entire length of boundaries in Fig. 1, $a$, parts $A B, C D$ of Fig. 1,b) and type II

* The flux $\Phi_{S}$ can be also chosen so that it is an integer multiple of $\Phi_{0}$ : this would not change significantly the final results but would change intermediate arguments and make them longer, so for clarity we discuss in detail only the half-integer case here. Note, however, that the main quantitative effect of this alternative choice of the flux is beneficial: it would push up the phase transition line separating the topological and superconducting phases shown in Fig. 3 for half integer case. 


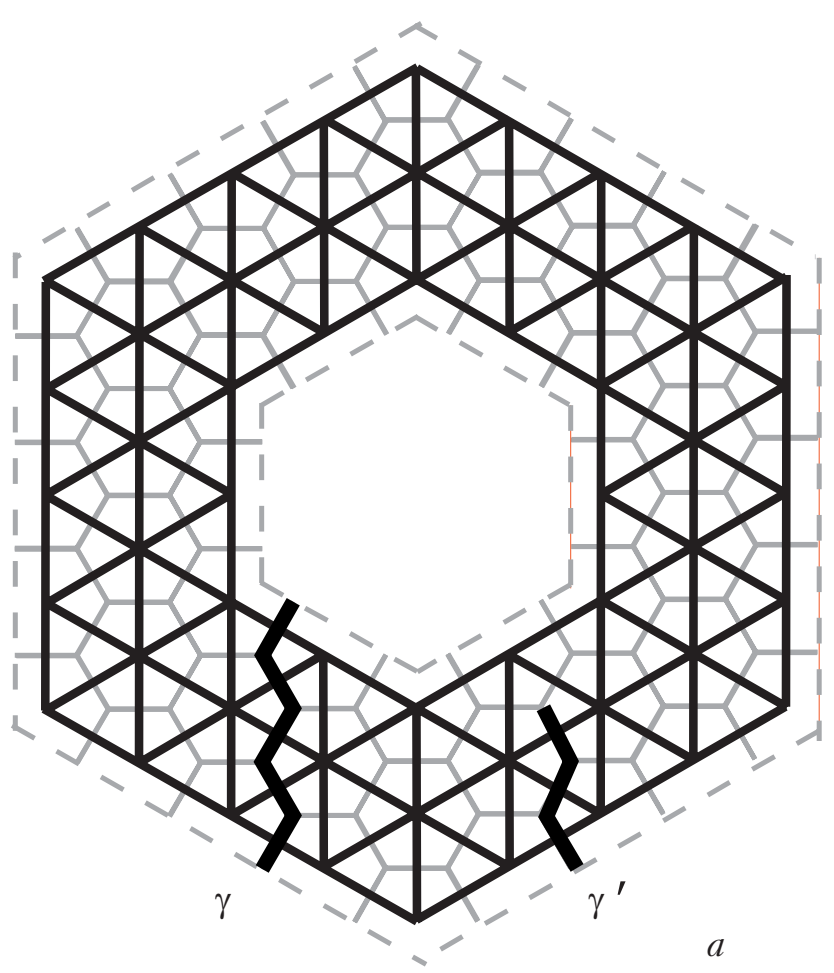

the entire boundary corresponds to one degree of freedom (phase at some point) while type II boundary includes many rhombi so it contains many degrees of freedom.

Note that each (inner and outer) boundary shown in Fig. 1, $a$ contains one rhombus; we included it to allow flux to enter and exit through the boundary when it is energetically favorable.

\section{Ground state, excitations and topological order}

In order to identify the relevant degrees of freedom in this highly frustrated system we consider first an individual rhombus. As a function of the gauge invariant phase difference between the far ends of the rhombus the potential energy is

$$
U\left(\varphi_{i j}\right)=-2 E_{J}\left(\left|\cos \left(\varphi_{i j} / 2\right)\right|+\left|\sin \left(\varphi_{i j} / 2\right)\right|\right) .
$$

This energy has two equivalent minima, at $\varphi_{i j}= \pm \pi / 2$ which can be used to construct elementary unprotected qubit, see [26]. In each of these states the phase changes by $\pm \pi / 4$ in each junction clockwise around the rhombus. We denote these states as $|\uparrow\rangle$ and $|\downarrow\rangle$ respectively. In the limit of large Josephson energy the space of low energy states of the full lattice is described by these binary degrees of freedom, the set of operators acting on these states is given by Pauli matrices $\sigma_{a b}^{x, y, z}$. We now combine these rhombi into hexagons forming the lattice shown in Fig. 1. This gives another condition: the sum of phase differences around the hexagon should equal to the flux $\Phi_{s}$ through each David's star inscribed in this hexagon. The choice $\varphi_{i j}=\pi / 2$ is consistent with flux $\Phi_{s}$ that is equal to a half integer number of flux quanta. This state minimizes the potential energy (2) of the system. This is, however, not the only choice. Although flipping the phase of one rhombus changes the phase flux around the star by $\pi$ and thus is prohibited, flipping two, four and six rhombi is allowed; generally the low energy configurations of $U(\varphi)$ satisfy the constraint

$$
\hat{P}_{a}=\prod_{b} \sigma_{a b}^{z}=1
$$

$\gamma$ and $\gamma^{\prime}$ are used in the construction of topological order parameter and excitations $(a)$. The lattice with $K=3$ openings, the ground state of Josephson junction array on this lattice is $2^{k}=8$ fold degenerate $(b)$.

boundary $(B C, A D)$. For both types of boundaries one needs to include in each boundary loop the flux which is equal to $1 / 4 Z_{b} \Phi_{0}$, where $Z_{b}$ is coordination number of the dual triangular lattice site. For instance, for the four coordinated boundary sites one needs to enclose the integer flux in these contours. In type I boundary

where the product runs over all neighbors, $b$, of site $a$. The number of (classical) states satisfying the constraint (3) is still huge: the corresponding configurational entropy is extensive (proportional to the number of sites). We now consider the charging energy of the contacts, which results in the quantum dynamics of the system. We show that it reduces this degeneracy to a much smaller number $2^{K}$. The dynamics of the individual rhombus is described by a simple Hamiltonian $H=\tilde{t} \sigma_{x}$ but the dynamics of a rhombus 
embedded in the array is different because individual flips are not compatible with the constraint (3). The simplest dynamics compatible with (3) contains flips of three rhombi belonging to the elementary triangle, $(a, b, c), \hat{Q}_{(a b c)}=\sigma_{a b}^{x} \sigma_{b c}^{x} \sigma_{c a}^{x}$ and therefore the simplest quantum Hamiltonian operating on the subspace defined by (3) is

$$
H=-r \sum_{(a b c)} Q_{(a b c)} .
$$

We discuss the derivation of the coefficient $r$ in this Hamiltonian and the correction terms and their effects below but first we solve the simplified model (3), (4) and show that its ground state is «protected» in the sense described above and that excitations are separated by the gap*.

Clearly, it is very important that the constraint is imposed on all sites, including boundaries. Evidently, some boundary hexagons are only partially complete but the constraint should be still imposed on the corresponding sites of the corresponding triangular lattice. This is ensured by additional superconducting wires that close the boundary hexagons in Fig. 1.

We note that constraint operators commute not only with the full Hamiltonian but also with individual $\hat{Q}_{(a b c)}:\left[\hat{P}_{a}, \hat{Q}_{(a b c)}\right]=0$. The Hamiltonian (4) without constraint has an obvious ground state, $|0\rangle$, in which $\sigma_{a b}^{x}=1$ for all rhombi. This ground state, however, violates the constraint. This can be fixed noting that since operators $\hat{P}_{a}$ commute with the Hamiltonian, any state obtained from $|0\rangle$ by acting on it by $\hat{P}_{a}$ is also a ground state. We can now construct a true ground state satisfying the constraint by

$$
|G\rangle=\prod_{a} \frac{1+\hat{P}_{a}}{\sqrt{2}}|0\rangle .
$$

Here $\left(1+\hat{P}_{a}\right) / \sqrt{2}$ is a projector onto the subspace satisfying the constraint at site $a$ and preserving the normalization.

Obviously, the Hamiltonian (4) commutes with any product of $\hat{P}_{a}$ which is equal to the product of $\sigma_{a b}^{z}$ operators around a set of closed loops. These integrals of motion are fixed by the constraint. However, for a topologically non-trivial system there appear a number of other integrals of motion. For a system with $K$ openings a product of $\sigma_{a b}^{z}$ operators along contour, $\gamma$ that begins at one opening and ends at another (or at the outer boundary, see Fig. 2)

$$
\hat{T_{q}}=\prod_{\left(\gamma_{q}\right)} \sigma_{a b}^{z}
$$

commutes with Hamiltonian and is not fixed by the constraint. Physically these operators count the parity of «up» rhombi along such contour. The presence of these operators results in the degeneracy of the ground state. Note that multiplying such operator by an appropriate $\hat{P}_{a}$ gives similar operator defined on the shifted contour so all topologically equivalent contours give one new integral of motion. Further, multiplying two operators defined along the contours beginning at the same (e.g., outer) boundary and ending in different openings, $A, B$ is equivalent to the operator defined on the contour leading from $A$ to $B$, so the independent operators can be defined (e.g.) by the set of contours that begin at one opening and ends at the outer boundary. The state $|G\rangle$ constructed above is not an eigenstate of these operators but this can be fixed defining

$$
\left|G_{f}\right\rangle=\prod_{q} \frac{1+c_{q} \hat{T}_{q}}{\sqrt{2}}|G\rangle,
$$

where $c_{q}= \pm 1$ is the eigenvalue of $\hat{T_{q}}$ operator defined on contour $\gamma_{q}$. Equation (7) is the final expression for the ground state eigenfunctions.

Construction of the excitations is similar to the construction of the ground state. First, one notices that since all operators $\hat{Q}_{a b c}$ commute with each other and with the constraints, any state of the system can be characterized by the eigenvalues $\left(Q_{a b c}= \pm 1\right)$ of $\hat{Q}_{a b c}$. The lowest excited state correspond to only one $Q_{a b c}$ being -1 . Notice that a simple flip of one rhombus (by operator $\sigma_{(a b)}^{z}$ somewhere in the system changes the sign of two $Q_{a b c}$ eigenvalues corresponding to two triangles to which it belongs. To change only one $Q_{a b c}$ one needs to consider a continuous string of these flip operators starting from the boundary: $|(a b c)\rangle=v_{(a b c)}|0\rangle$ with $v_{(a b c)}=\prod_{\gamma^{\prime}} \sigma_{(c d)}^{z}$, where the product is over all rhombi $(c d)$ that belong to the path $\gamma^{\prime}$ that begins at the boundary and ends at $(a b c)$ (see Fig. 2, which shows one such path). This operator changes the sign of only one $Q_{a b c}$, the one that corresponds to the «last» triangle. This construction does not satisfy the constraint, so we have to apply the same «fix» as for the ground state construction above

* In a rotated basis $\sigma^{x} \rightarrow \sigma^{z}, \sigma^{z} \rightarrow \sigma^{x}$ this model is reduced to a special case of $Z_{2}$ lattice gauge theory [27,28] which contains only magnetic term in the Hamiltonian with the constraint (3) playing a role of a gauge invariance condition. 


$$
\left|v_{(a b c)}\right\rangle=\prod_{q} \frac{1+c_{q} \hat{T}_{q}}{\sqrt{2}} \prod_{a} \frac{1+\hat{P}_{a}}{\sqrt{2}} v_{(a b c)}|0\rangle
$$

to get the final expression for the lowest energy excitations. The energy of each excitation is $2 r$. Note that a single flip excitation at a rhombus $(a b)$ can be viewed as a combination of two elementary excitations located at the centers of the triangles to which rhombus ( $a b)$ belongs and has twice their energy. Generally, all excited states of the model (4) can be characterized as a number of elementary excitations (8), so they give exact quasiparticle basis. Note that creation of a quasiparticle at one boundary and moving it to another is equivalent to the $\hat{T}_{q}$ operator, so this process acts as $\tau_{q}^{z}$ in the space of the $2^{K}$ degenerate ground states. As will be shown below, in the physical system of Josephson junctions these excitations carry charge $2 e$ so that $\tau_{q}^{z}$ process is equivalent to the charge $2 e$ transfer from one boundary to another.

Consider now the matrix elements, $O_{\alpha \beta}=\left\langle G_{\alpha}|\hat{O}| G_{\beta}\right\rangle$ of a local operator, $\hat{O}$, between two ground states, e.g., of an operator that is composed of a small number of $\sigma_{a b}$. To evaluate this matrix element we first project a general operator onto the space that satisfies the constraint: $\hat{O} \rightarrow \mathcal{P O} \mathcal{P}$, where $\mathcal{P}=\prod_{a}\left(1+\hat{P}_{a}\right) / 2$. The new (projected) operator is also local, it has the same matrix elements between ground states but it commutes with all $\hat{P}_{a}$. Since it is local it can be represented as a product of $\sigma_{z}$ and $\hat{Q}$ operators which implies that it also commutes with all $\hat{T}_{q}$. Thus, its matrix elements between different states are exactly zero. Further, using the fact that it commutes with $\hat{P}_{a}$ and $\hat{T}_{q}$ we write the difference between its diagonal elements evaluated between the states that differ by a parity over contour $q$ as

$$
O_{+}-O_{-}=\left\langle 0\left|\prod_{i} \frac{1+\hat{P}_{i}}{\sqrt{2}} \hat{T_{q}} \hat{O}\right| 0\right\rangle .
$$

This equation can be viewed as a sum of products of $\sigma_{z}$ operators. Clearly to get a non-zero contribution each $\sigma^{z}$ should enter even number of times. Each $\hat{P}$ contains a closed loop of six $\sigma^{z}$ operators, so any product of these terms is also a collection of a closed loops of $\sigma^{z}$. In contrast to it, operator $\hat{T}_{q}$ contains a product of $\sigma^{z}$ operators along the loop $\gamma$, so the product of them contains a string of $\sigma_{z}$ operators along the contour that is topologically equivalent to $\gamma$. Thus, one gets a non-zero $\mathrm{O}_{+}-\mathrm{O}_{-}$only for the operators $\hat{O}$ which contain a string of $\sigma^{z}$ operators along the loop that is topologically equivalent to $\gamma$ which is impossible for a local operator. Thus, we conclude that for this model all non-diagonal matrix elements of a local operator are exactly zero while all diagonal are exactly equal.

\section{Effect of physical perturbations}

We now come back to the original physical system described by the Lagrangian (1) and derive the parameters of the model (4) and discuss the most important corrections to it and their effect. We begin with the derivation. In the limit of small charging energy the flip of three rhombi occurs by a virtual process in which the phase, $\varphi_{i}$ at one (6-coordinated) island, $i$, changes by $\pi$. In the quasiclassical limit the phase differences on the individual junctions are $\varphi_{\text {ind }}= \pm \pi / 4$; the leading quantum process changes the phase on one junction by $3 \pi / 2$ and on others by $-\pi / 2$ changing the phase across the rhombus $\varphi \rightarrow \varphi+\pi$. The phase differences, $\varphi$, satisfy the constraint that the sum of them over the closed loops remain $2 \pi\left(n+\Phi_{s} / \Phi_{0}\right)$. The simplest such process preserves the symmetry of the lattice, and changes simultaneously the phase differences on the three rhombi containing island $i$ keeping all other phases constant. The action for such process is three times the action of elementary transitions of individual rhombi, $S_{0}$ :

$$
r \approx E_{J}^{3 / 4} E_{C}^{1 / 4} \exp \left(-3 S_{0}\right), \quad S_{0}=1.61 \sqrt{E_{J} / E_{C}} .
$$

In the alternative process the phase differences between $i$ and other islands change in turn, via high energy intermediate state in which one phase difference has changed while others remained close to their original values. The estimate for this action shows that it is larger than $3 S_{0}$, so (10) gives the dominating contribution. There are in fact many processes that contribute to this transition: the phase of island $i$ can change by $\pm \pi$ and, in addition, in each rombus one can choose arbitrary the junction in which the phase changes by $\pm 3 \pi / 2$; the amplitude of all these processes should be added. This does not change the result qualitatively unless these amplitudes exactly cancel each other, which happens only if the charge of the island is exactly half-integer (because phase and charge are conjugate the amplitude difference of the processes that are different by $2 \pi$ is $\exp (2 \pi i q))$. We assume that in a generic case this cancellation does not occur. External electrical fields (created by, e.g., stray charges) might induce non-integer charges on each island which would lead to a randomness in the phase and amplitude of $r$. The phase of $r$ can be eliminated by a proper gauge transformation 
$|\uparrow\rangle_{a b} \rightarrow \exp \left(i \alpha_{a b}\right)|\uparrow\rangle_{a b}$ and has no effect at all. The amplitude variations result in a position dependent quasiparticle energy.

We now consider the corrections to the model (4). One important source of corrections is the difference of the actual magnetic flux through each rhombus from the ideal value $\Phi_{0} / 2$. If this difference is small it leads to the bias of «up» versus «down» states, their energy difference becomes

$$
2 \epsilon=2 \pi \sqrt{2} \frac{\delta \Phi_{d}}{\Phi_{0}} E_{J} .
$$

Similarly, the difference of the actual flux through David's star and the difference in the Josephson energies of individual contacts leads to the interaction between «up» states:

$$
\delta H_{1}=\sum_{(a b)} V_{a b} \sigma_{a b}^{z}+\sum_{(a b),(c d)} V_{(a b),(c d)} \sigma_{a b}^{z} \sigma_{c d}^{z},
$$

where $V_{a b}=\epsilon$ for uniform field deviating slightly from the ideal value and $V_{(a b),(c d)} \neq 0$ for rhombi belonging to the same hexagon. Consider now the effect of perturbations described by $\delta H_{1}$, Eq. (12). These terms commute with the constraint but do not commute with the main term, $H$, so the ground state is no longer $\left|G_{ \pm}\right\rangle$. In other words, these terms create excitations (8) and give them kinetic energy. In the leading order of the perturbation theory the ground state becomes $\left|G_{ \pm}\right\rangle+(\epsilon / 4 r) \sum_{(a b)} \sigma_{(a b)}^{z}\left|G_{i \pm}\right\rangle$. Qualitatively, it corresponds to the appearance of virtual pairs of quasiparticles in the ground state. The density of these quasiparticles is $\epsilon / r$. As long as these quasiparticles do not form a topologically non-trivial string all previous conclusions remain valid. However, there is a nonzero amplitude to form such a string, it is now exponential in the system size. With exponential accuracy this amplitude is $(\epsilon / 2 r)^{L}$ which leads to an energy splitting of the two ground state levels and the matrix elements of typical local operators of the same order

$$
E_{+}-E_{-} \sim O_{+}-O_{-} \sim(\epsilon / 2 r)^{L} .
$$

The physical meaning of the $v_{(a b c)}$ excitations becomes more clear if one consider the effect of the addition of one $\sigma^{z}$ operator to the end of the string defining the quasiparticle: it results in the charge transfer of $2 e$ across this last rhombus. To prove this, note that the wave function of a superconductor corresponding to the state which is symmetric combination of $|\uparrow\rangle$ and $|\downarrow\rangle$ is periodic with period $\pi$ and thus corresponds to charge which is multiple of $4 e$ while the antisymmetric corresponds to charge $(2 n+1) 2 e$. The action of one $\sigma_{z}$ $\sigma_{z}$ induces the transition between these states and thus transfers the charge $2 e$. Thus, these excitations carry charge $2 e$. Note that continuous degrees of freedom are characterized by the long range order in $\cos (2 \varphi)$ and thus correspond to the condensation of pairs of Cooper pairs. In other words, this system superconducts with elementary charge $4 e$ and has a gap, $2 r$, to the excitations carrying charge $2 e$. Similar pairing of Cooper pairs was shown to occur in a chain of rhombi in a recent paper [29]; formation of a classical superconductive state with effective charge $6 e$ in frustrated Kagome wire network was predicted in [30].

The model (4) completely ignores the processes that violate the constraint at each hexagon. Such processes might violate the conservation of the topological invariants $\hat{T}_{q}$ and thus are important for a long time dynamics of the ground state manifold. In order to consider these processes we need to go back to the full description involving the continuous superconducting phases $\varphi_{i}$. Since potential energy (2) is periodic in $\pi$ it is convenient to separate the degrees of freedom into continuous part (defined modulus $\pi$ ) and discrete parts. Continuous parts have a long range order: $<\cos \left(2 \varphi_{0}-2 \varphi_{r}\right)>\sim 1$. The elementary excitations of the continuous degrees of freedom are harmonic oscillations and vortices. The harmonic oscillations interact with discrete degrees of freedom only through the local currents that they generate. Further the potential (2) is very close to the quadratic, so we conclude that they are practically decoupled from the rest of the system. In contrast to this vortices have an important effect. By construction, the elementary vortex carries flux $\pi$ in this problem. Consider the structure of these vortices in a greater detail. The superconducting phase should change by 0 or $2 \pi$ when one moves around a closed loop. In a half vortex this is achieved if the gradual change by $\pi$ is compensated (or augmented) by a discrete change by $\pi$ on a string of rhombi which costs no energy. Thus, from the viewpoint of discrete degrees of freedom the position of the vortex is the hexagon where constraint (3) is violated. The energy of the vortex is found from the usual arguments

$$
E_{v}(R)=\frac{\pi E_{J}}{4 \sqrt{6}}(\ln R+c), c \approx 1.2 ;
$$

it is logarithmic in the vortex size, $R$. The process that changes the topological invariant $\hat{T}_{q}$ is the one in which one half vortex completes a circle around an opening. The amplitude of such process is exponentially small: $\left(\tilde{t} / E_{v}(D)\right)^{\Lambda}$, where $\tilde{t}$ is the amplitude to flip one rhombus and $\Lambda$ is the length of the shortest path around the opening. In the quasiclassical limit the amplitude $\tilde{t}$ can be estimated analogously to (10): 


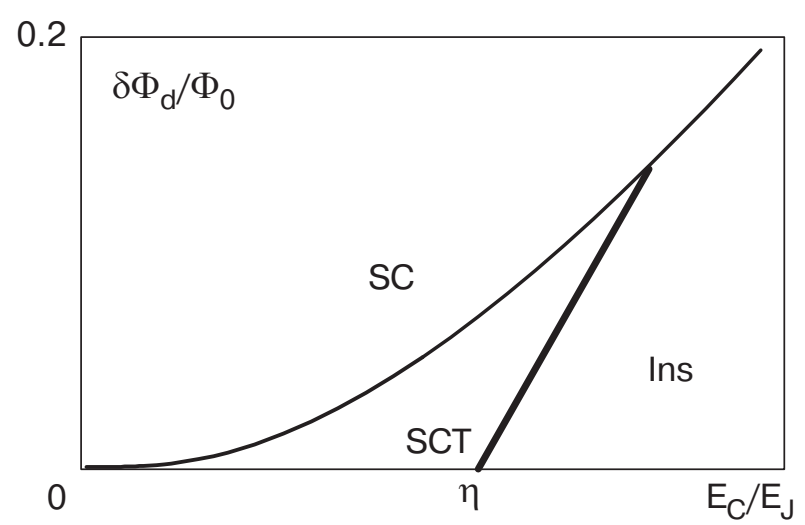

Fig. 3. Schematic of the phase diagram for half integer $\Phi_{S}$ at low temperatures: $\delta \Phi_{d}$ is the deviation of the magnetic flux through each rhombus from its ideal value. SC stands for usual superconducting phase, SCT for the phase with $\cos (2 \varphi)$ long range order of the continuous degrees of freedom and discrete topological order parameter discussed extensively in the bulk of the paper. The SCT phase and SC phase are separated by 2D quantum Ising phase transition.

$\tilde{t} \sim \sqrt{E_{J} E_{Q}} \exp \left(-S_{0}\right)$. The half vortices would appear in a realistic system if the flux through each hexagon is systematically different from the ideal half integer value. The presence of free vortices destroys topological invariants, so a realistic system should either be not too large (so that deviations of the total flux do not induce free vortices) or these vortices should be localized in prepared traps (e.g., David's stars with fluxes slightly larger or smaller than $\Phi_{s}$ ). If the absence of half vortices the model is equivalent to the Kitaev model [7] placed on triangular lattice in the limit of the infinite energy of the excitation violating the constraints.

Quantitatively, the expression for the parameters of the model (4) becomes exact only if $E_{J} \gg E_{C}$. One expects, however, that the qualitative conclusions remain the same and the formulas derived above provide reliable estimates of the scales even for $E_{J} \sim E_{C}$, provided that charging energy is not so large as to result in a phase transition to a different phase. One expects this transition to occur at $E_{C}^{*}=\eta E_{J}$ with $\eta \sim 1$ which exact value can be reliably determined only from numerical simulations. Practically, since the perturbations induced by flux deviations from $\Phi_{0}$ are proportional to $\left(\delta \Phi / \Phi_{0}\right) \times$ $\times\left(E_{J} / r\right)$ and $r$ becomes exponentially small at small $E_{C}$, the optimal choice of the parameters for the physical system is $E_{C} \approx E_{C}^{*}$. We show the schematics of the phase diagram in Fig. 3. We assume here that transition to insulating phase is direct, another alternative is the intermediate phase in which the energy of the vortex becomes finite instead of being logarithmic. If this phase indeed exists it is likely to have properties more similar to the one discussed in [7] (in the next
Section we consider generalized $J J$ array, where such am intermediate phase does exist). The «topological» phase is stable in a significant part of the phase diagram. The phase transition between «topological superconductor» and usual superconductor belongs to the class of quantum spin-1/2 2D Ising model on a hexagonal lattice, placed in a transverse field:

$$
H_{\text {Ising }}=-\epsilon \sum_{(i j)} s_{j}^{x} s_{i}^{x}-r \sum_{i} s_{i}^{z} .
$$

Here $i, j$ denote sites of hexagonal lattice, eigenvalue of operator $s_{i}^{z}$ measures parity of Cooper pairs number $n_{i}$ on the $i$ th island: $n_{i}(\bmod 2)=1 / 2\left(1-s_{i}^{z}\right)$; parameters $r$ and $\epsilon$ are defined in Eqs. (10), (11). As long as the ratio $\lambda=r / \epsilon$ is larger than some critical value $\lambda_{c}$, ground state obeys even number of Cooper pairs on each island, which corresponds to our «topological superconductor» phase. The values of $\lambda_{c}$ for square, triangular and cubic lattices was found via quantum Monte-Carlo simulations [31]; in particular, $\lambda_{c}^{\text {triang }} \approx 4.6 \pm 0.3$, and $\lambda_{c}^{\text {square }} \approx 2.7 \pm 0.3$. There is no available data for a hexagonal lattice; based upon above-cited results one could estimate $\lambda_{c}^{\text {hex }} \approx 2 \pm 0.5$.

Furthermore, since the vortex excitations have logarithmic energy, we expect that this phase survives at finite temperatures as well. In the thermodynamic limit, at $T \neq 0$ one gets a finite density of $2 e$ carrying excitations $\left(n_{v} \sim \exp (-2 r / T)\right)$ but the vortices remain absent as long as temperature is below BKT-like depairing transition for half-vortices.

\section{Aray 2: Topological insulator}

Generally, increasing the charging energy in a Josephson junction array makes it an insulator. This transition is due to an increase of phase fluctuations in the original array and the resulting appearance of free vortices that form a superfluid of their own. The new situation arises in topological superconductor because it allows half-vortices. Two scenarios are now possible. The «conventional» scenario would involve condensation of half-vortices since they are conjugate to $4 e$ charges. In this case we get an insulator with elementary excitations carrying charge $4 e$. An alternative is condensation of full vortices (pairs of half vortices) with a finite gap to half vortices. In this case the elementary excitations are charge $2 e$ objects. Similar fractionalization was discussed in the context of high $T_{c}$ superconductors in $[19,32]$ and in the context of spin or quantum dimer systems in [22,33-36,]. Such insulator acquires interesting topological properties on a lattice with holes because each hole leads to a new binary degree of freedom which describes presence or absence of half vortex. The energies of these 


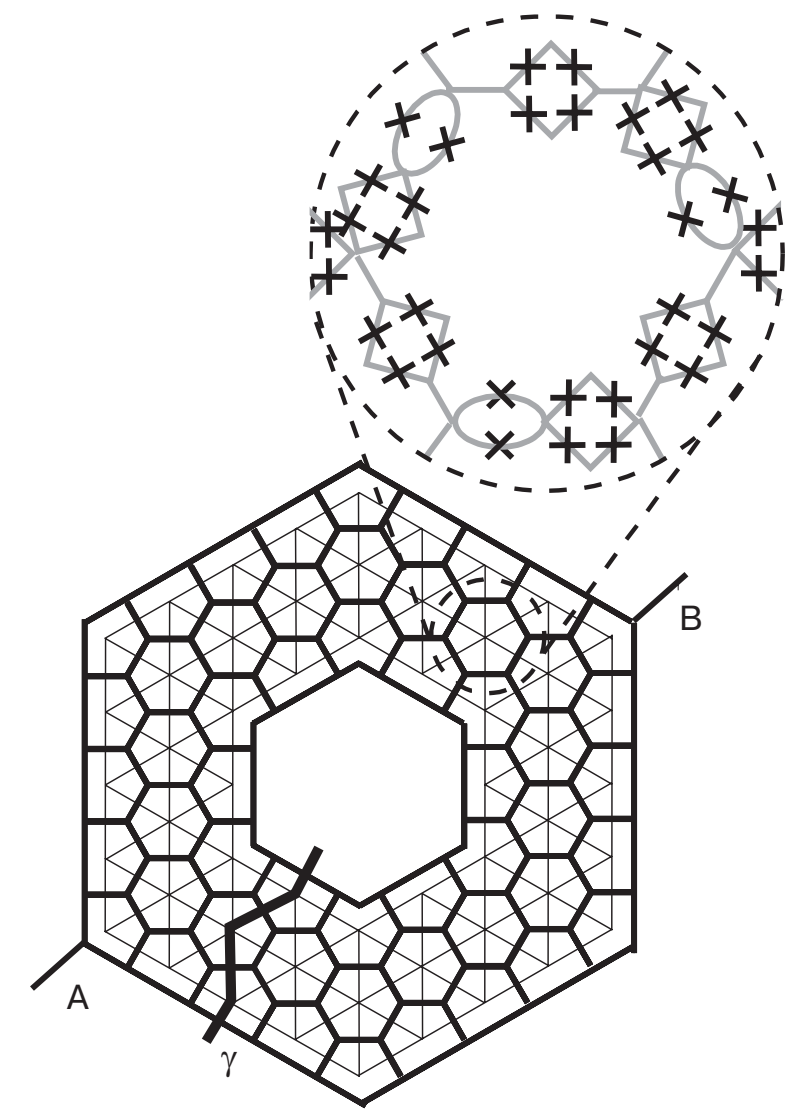

Fig. 4. Schematics of the array. The main figure: Global structure of the array. Discrete variables controlling the low energy properties are defined on the links of the hexagonal lattice. Generally, the lattice might have $K$ big holes, here we show $K=1$ example. Zoom in: Each inner bond of the lattice contains a rhombus made out of four Josephson junctions; some bonds also contain an effective weaker link made of two Josephson junctions so that each hexagon of the lattice contains $k=3$ such links. The flux through each rhombus is half-flux quanta, $\Phi_{0} / 2$, the flux through a loop constituting a weak link is close to half flux quanta $\Phi=\Phi_{0} / 2+\delta \Phi$. The boundary of the lattice contains rhombi and weak links so that each boundary plaquette has the same number, $k$, of weak links as the bulk hexagon.

states are equal up to corrections which vanish exponentially with the size of the holes. These states cannot be distinguished by local measurements and have all properties expected for a topological insulator. They can be measured, however, if the system is adiabatically brought into the superconductive state by changing some controlling parameter. Here we propose a modification of the «topologically superconductive» array that provides such control parameter and, at the same time, allows us to solve the model and compute the properties of the topological insulator. The key idea of this modification is to allow full (of flux $\Phi_{0}$ ) vortices move with large amplitude be- tween plackets of the hexagonal lattice, so that they lower their energy due to delocalization, and eventually Bose-condense, while half-vortices are still kept (almost) localized.

Consider array shown in Fig. 4 that contains rhombi with junctions characterized by Josephson and charging energies $E_{J}>E_{C}$ and weak junctions with $\epsilon_{J}<<\epsilon_{C}<<E_{C}$. Each rhombus encloses half of a flux quantum leading to an exact degeneracy between the two states of opposite chirality of the circulating current $[13,29]$. This degeneracy is a consequence of the symmetry operation which combines the reflection about the long diagonal of the rhombus and a gauge transformation needed to compensate the change of the flux $\Phi_{0} / 2 \rightarrow-\Phi_{0} / 2$. This gauge transformation changes the phase difference along the diagonal by $\pi$. This $z_{2}$ symmetry implies the conservation of the parity of the number of pairs at each site of the hexagonal lattice and is the origin of the Cooper pair binding. We assume that each elementary hexagon contains exactly $k$ such junctions: in case each link contains one weak junction $k=6$, but generally it can take any value $k \geq 1$. As will be shown below, the important condition is the number of weak junctions that one needs to cross in the elementary loop. Qualitatively, a value $k \geq 1$ ensures that it costs a little to put vortex in any hexagon.

For the general arguments that follow below the actual construction of the weak links is not important, however, practically it is difficult to vary the ratio of the capacitance to the Josephson energy so weaker Josephson contact usually implies larger Coulomb energy. This can be avoided if weak contact is made from Josephson junction loop frustrated by magnetic field. The charging energy of this system is half the charging energy of the individual junction while the effective Josephson junction strength is $\epsilon_{J}=2 \pi\left(\delta \Phi / \Phi_{0}\right) E_{0}$, where $E_{0}$ is the Josephson energy of each contact and $\delta \Phi=\Phi-\Phi_{0} / 2$ is the difference of the flux from half flux quanta. This construction also allows to control the system under varying the magnetic field.

In these conditions the whole array is insulating. Assume that $\epsilon_{J}$ sets the lowest energy scale in this problem (the exact condition will be discussed below). The state of the array is controlled by discrete variables $u_{i j}=0,1$ which describe the chiral state of each rhombus and by continuous phases $\varphi_{i j}$ that specify the state of each weak link (here and below $i, j$ denote the sites of hexagonal lattice). If Josephson coupling $\epsilon_{J} \equiv 0$, different islands are completely decoupled and potential energy does not depend on discrete variables $u_{i j}$. For small $\epsilon_{J}$ we can evaluate its effect in the perturbation theory: 


$$
V(u)=-W \cos \left(\pi \sum_{\text {hex }} u_{i j}\right), \quad W=\frac{k^{k}}{k !} \epsilon_{J}\left(\frac{\epsilon_{J}}{8 \epsilon_{C}}\right)^{k-1} .
$$

This potential energy lowers the relative energy of classical configurations of $u_{i j}$ that satisfy the constraint $\sum_{\text {hex }} u \equiv 0(\bmod 2)$ but it does not prohibit configurations with $\sum_{\text {hex }} u \equiv 1(\bmod 2)$.

Consider now the dynamics of discrete variables. Generally, two types of tunneling processes are possible. In the first type the phase changes by $\pi$ across each of the three rhombi that have a common site. This is the same process that gives the leading contribution to the dynamics of the superconducting array [13], its amplitude is given (in the quasiclassical approximation) by Eq. (10) above. In the second type of process the phase changes across one rhombus and across one weak junction. Because the potential energy of the weak junction is assumed to be very small the main effect of the weak junction is to change the kinetic energy. The total kinetic energy for this process is the sum of the terms due to the phase across the rhombi and across the weak link. Assuming that these phase variations are equal and opposite in sign, the former is about $E_{C}^{-1} \varphi^{2}$ while the latter $\epsilon_{C}^{-1} \varphi^{2}$, so the effective charging energy of this process is $\tilde{E}_{C}=\left(E_{C}^{-1}+\epsilon_{C}^{-1}\right)^{-1}$. For $\epsilon_{C} \ll E_{C}$ this charging energy is small and such process is suppressed. Thus, in these conditions the dominating process is the simultaneous flip of three rhombi as in the superconducting case. In the following we restrict ourselves to this case. Further, we shall assume that $r>W$ so that in the leading order one can neglect the potential energy compared to the kinetic energy corresponding to the flip of three rhombi. As $W$ is increased by turning on $\epsilon_{J}$ the continuous phase $\varphi_{i j}$ orders and the transition into the superconducting state happens at $\epsilon_{J} \sim \epsilon_{C}$. At larger $\epsilon_{J}, W$ becomes $\epsilon_{J}$ and with a further increase of $\epsilon_{J}$, for $\epsilon_{J} \gg r$ vortices completely disappear from the low energy spectrum and the array becomes equivalent to the one studied in [13].

The low energy states are the ones that minimize the kinetic energy corresponding to simultaneous flip processes:

$$
H_{T}=-r \sum_{i} \prod_{j(i)} \tau_{i j}^{x}
$$

Here $j(i)$ denote the nearest neighbors of site $i, \tau_{i j}^{x}$ is the operator that flips discrete variables $u_{i j}$ and $r$ is given by (10). The states minimizing this energy satisfy the gauge invariance condition

$$
\prod_{j(i)} \tau_{i j}^{x}|\Psi\rangle=|\Psi\rangle .
$$

The Hilbert space of states that satisfy the condition (17) is still huge. If all weaker terms in the Hamiltonian are neglected all states that satisfy (17) are degenerate. These states can be visualized in terms of half vortices positioned on the sites of the dual lattice, $a, b$. Indeed, a convenient way to describe different states that satisfy (17) is to note that operator $\Pi_{j(i)} \tau_{i j}^{x}$ does not change the value of $\sum_{\mathrm{hex}} u_{i j}$ for second type of tunneling processes. Thus, one can fix the values of $\sum_{\text {hex }} u_{i j}=v_{a}$ on all hexagonal plaquettes, $a$ and impose the constraint (17). In physical terms the binary values $v_{i}=0,1$ describe the positions of half-vortices on dual (triangular) lattice. This degeneracy between different states is lifted when the subdominant terms are taken into account. The main contribution to the potential energy of these half-vortices comes from (15), it is simply proportional to their number. The dynamics of these vortices is due to the processes in which only one rhombus changes its state and the corresponding flip of the phase accross the weak junction. The amplitude of this process is

$$
\tilde{r} \approx E_{J}^{3 / 4} E_{C}^{1 / 4} \exp \left(-\tilde{S_{0}}\right), \quad \tilde{S_{0}}=1.61 \sqrt{\frac{E_{J}}{\tilde{E_{C}}}} .
$$

In this approximation the effective Hamiltonian controlling these vortices becomes (cf., Eq. (14)):

$$
H_{v}=-\tilde{r} \sum_{(a b)} \sigma_{a}^{x} \sigma_{b}^{x}-W \sum_{a} \sigma_{a}^{z}
$$

where operators $\sigma_{a}$ act in the usual way on the states with/without half-vortices at plaquette $a$ and the first sum runs over adjacent plaquettes $(a b)$. This Hamiltonian describes an Ising model in a transverse field. For small $W / \tilde{r}<\lambda_{c} \sim 1$ its ground state is «disordered»: $\left\langle\sigma^{z}\right\rangle=0$ but $\left\langle\sigma^{x}\right\rangle \neq 0$ while for $W / \tilde{r}>\lambda_{c}$ it is «ordered»: $\left\langle\sigma^{z}\right\rangle \neq 0,\left\langle\sigma^{x}\right\rangle=0$. The critical value of transverse field is known from extensive numerical simulations [31]: $\lambda_{c} \approx 4.6 \pm 0.3$ for triangular lattice. The «disordered» state corresponds to the liquid of half-vortices, while in the «ordered» state the density of free half-vortices vanishes, i.e., the ground state contains even number of half-vortices so the total vorticity of the system is zero. To prove this we start from the state $|\uparrow\rangle$ which is the ground state at $\tilde{r} / W=0$ and consider the effect of $\tilde{r} \sum_{(a b)} \sigma_{b}^{x} \sigma_{a}^{x}$ in perturbation theory. Higher energy states are separated from the ground state by the gap $W$ so each order is finite. Further in each order operator $\sigma_{a}^{x} \sigma_{b}^{x}$ creates two more half-vortices proving that the total number of half-vortices remains even in each order. 
Table

The typical properties of topological superconductor and insulator

\begin{tabular}{|c|c|c|}
\hline State & Topological superconductor & Topological insulator \\
\hline Ground state & Condensate of $4 e$ charges & Condensate of $2 \pi$ phase vortices \\
\hline Fluxons & Gapful, charge $2 e$ & Gapful, $\pi$ phase vortices \\
\hline Pseudocharges & Half fluxes with energy $\epsilon \sim E_{J} \log L$ & Charge $2 e$ with $\epsilon=2 r+\beta \epsilon_{C} \min \left[\log L, \log \left(c / c_{0}\right)\right]^{\star}$ \\
\hline Ground state degeneracy & Charge on the inner boundary mod $4 e$ & Number of $\pi$ vortices inside the hole $\bmod 2$ \\
\hline Ground state splitting & $\left(\delta \Phi / \Phi_{0}\right)\left(E_{J} / r\right)^{L}$ & $(\tilde{r} / W)^{L}$ \\
\hline
\end{tabular}

$\mathrm{C} \mathrm{o} \mathrm{m} \mathrm{m} \mathrm{e} \mathrm{t}$ : ${ }^{*}$ here $\beta$ is the number $\sim 1, c$ is the capacitance of a weak link and $c_{0}$ is the self-capacitance of an island.

The states with odd number of half-vortices have a gap $\Delta(\tilde{r} / W)$ which remains non-zero for $W / \tilde{r}>\lambda_{c}$.

In terms of the original discrete variable defined on the rhombi the Hamiltonian (18) becomes

$$
H_{u}=-\tilde{r} \sum_{(i j)} \tau_{i j}^{x}-W \sum_{i} \prod_{j(i)} \tau_{i j}^{z},
$$

where $\tau$-operators act on the state of each rhombus. This Hamiltonian commutes with the constraint (17) and is in fact the simplest Hamiltonian of the lattice $z_{2}$ gauge theory. The disordered regime corresponds to a confined phase of this $z_{2}$ gauge theory, leading to elementary $4 e$ charge excitations and the ordered regime to the deconfined phase.

Consider now the system with non-trivial topology, e.g., a hole. In this case the set of variables $v_{a}$ is not sufficient to determine uniquely the state of the system, one has to supplement it by the variable $v_{0}=\Sigma_{L} u_{a b}$, where sum is taken over a closed contour $L$ that goes around the hole. Physically, it describes the presence/absence of the half-vortex in the hole. The effective Hamiltonian of this additional variable has only kinetic part because presence or absence of half vortex in a hole which has $l$ weak links in its perimeter gives potential energy $W_{0}=c \epsilon_{J}\left(\epsilon_{J} / \epsilon_{C}\right)^{l}$ which is exponentially small for $l>>1$. The kinetic part is similar to other variables: $H_{0}=-\tilde{r} \sum_{a \in I} \sigma_{a}^{x} \sigma_{0}^{x}$, it describes a process in which half-vortex jumps from the hole into the inner boundary, $I$, of the system. In the state with $\left\langle\sigma^{z}\right\rangle \neq 0$ this process increases the energy of the system by $\tilde{W}(\tilde{r} / W)(\tilde{W}(0)=W$ and $\left.\tilde{W}\left(\lambda_{c}\right)=0\right)$. In the state with $\left\langle\sigma^{x}\right\rangle \neq 0$ it costs nothing. Thus, the process in which half-vortex jumps from the hole into the system and another half-vortex exits into the outside region appears in the second order of the perturbation theory, the amplitude of this process is $t_{v}=\tilde{r}^{2} \sum_{i \in I, j \in O} g_{a b}$, where sum is performed over all sites of the inner $(I)$ and outer $(O)$ boundaries and

$$
g_{a b}=\left\langle\sigma_{a}^{x} \frac{1}{H-E_{0}} \sigma_{b}^{x}\right\rangle
$$

has a physical meaning of the half vortex tunneling amplitude from inner to outer boundaries. At small $\tilde{r} / W$, deep in the insulating phase, we can estimate $g_{a b}$ using the perturbation expansion in $\tilde{r} / W$ : the leading contribution appears in $|a-b|$ th order of the perturbation theory, thus $g_{a b} \propto(\tilde{r} / W)^{|a-b|}$. Thus for small $\tilde{r} / W$ the tunneling amplitude of the half vortex is exponentially small in the distance, $L$, from the outer to the inner boundary; we expect that it remains exponentially small for all $\tilde{r} / W<\lambda_{c}$. For $\tilde{r} / W>\lambda_{c}$ this amplitude is of the order of $\tilde{r}^{2} / W$ and therefore is significant.

In a different language, in the system with a hole we can construct a topological invariant $\mathcal{P}=\prod_{\gamma} \tau_{i j}^{x}$ (contour $\gamma$ is shown in Fig. 4) which can take values \pm 1 . Note that now the contour goes via triangular lattice sites (where vortices are defined), whereas in the first (superconductive array) version the corresponding path was drawn via sites of basic hexagonal lattice. The same arguments as used for the superconducting array show that any dynamics consistent with constraint (17) preserves $\mathcal{P}$. Thus, formally, the properties of the topological insulator are very similar to the properties of the topological superconductor discussed in [12], if one replaces the words Cooper pair by half-vortex and vice versa. We summarize this duality in the table.

Note that at small $\tilde{r} / W \rightarrow 0$ the ground state of the Hamiltonian (19) satisfies the condition (17) and minimizes the second term in (19), i.e., satisfies the condition $\Pi_{j(i)} \tau_{i j}^{z}|\Psi\rangle=|\Psi\rangle$; it can be explicitly written as

$$
|0\rangle_{\mathrm{ins}}=\prod_{i} \frac{1}{2}\left(1+\prod_{j(i)} \tau_{i j}^{z}\right) \prod_{k l}|\rightarrow\rangle_{k l} .
$$


This state is a linear superposition of the degenerate states with $P=1$ and $P=-1$ :

$$
|0\rangle_{\text {ins }}=\frac{1}{\sqrt{2}}\left(|+\rangle_{\text {ins }}+|-\rangle_{\text {ins }}\right)
$$

and it coincides with the ground state $|G\rangle$ of discrete variables in the superconducting array (cf., Eq.(5)). The orthogonal superposition of $P= \pm 1$ states,

$$
|1\rangle_{\text {ins }}=\frac{1}{\sqrt{2}}\left(|+\rangle_{\text {ins }}-|-\rangle_{\text {ins }}\right),
$$

corresponds to the half-vortex inside the hole. The energy difference between the above two states $E_{1}-E_{0}$ is exponentially small in the insulating state of the array, whereas it is large in the superconductive state.

\section{Quantum manipulations}

We now discuss the manipulation of the protected states formed in this system. We start from superconductive version of array.

First, we note that here the topological invariant $\hat{T}_{q}$ has a simple physical meaning - it measures the total phase difference (modulus $2 \pi$ ) between the inner and outer boundaries. In an array with even number of rhombi between internal and outer boundaries, the state with eigenvalue $c_{q}=1$ has phase difference 0 , whereas the state with eigenvalue $c_{q}=-1$ has phase difference $\pi$. It means that measuring this phase difference measures the state of the qubit in the same basis where $T_{q}$ is diagonal. For the following discussion we define a set of Pauli matrices $\Sigma_{q}^{x, y, z}$ acting in the $2 \times 2$ qubit space, such that $\Sigma_{q}^{z} \equiv T_{q}$.

Introducing a weak coupling between these boundaries by a very weak Josephson circuit (characterized by a small energy $e_{J}$ ) would change the phase of these states in a controllable manner, e.g., in a unitary transformation

$$
U^{z}=\exp \left(i e_{J} t \Sigma_{q}^{z}\right) .
$$

The transformation coupling two qubits can be obtained if one introduces a weak Josephson circuit that connects two different inner boundaries (corresponding to different qubits). Namely, it will produce operation

$$
U_{p, q}^{z}=\exp \left(i e_{J} t \Sigma_{q}^{z} \Sigma_{p}^{z}\right) .
$$

Analogously, the virtual process involving half vortex motion around the opening gives the tunneling amplitude, $\epsilon_{t}$ between topological sectors, e.g., unitary transformation $U^{x}=\exp \left(\right.$ it $\left.\epsilon_{t} \Sigma_{q}^{x}\right)$. This tunneling can be controlled by magnetic field if the system is prepared with some number of vortices that are pinned in the idle state in a special plaquette, where the flux is integer. The slow (adiabatic) change of this flux to towards a normal (half-integer) value would release the vortex and result in the transitions between topological sectors with $\epsilon_{t} \sim \tilde{t} / D^{2}$.

These operations are analogous to usual operations on a qubit and are prone to usual source of errors. This system, however, allows another type of operation that are naturally discrete. As we show above the transmission of the elementary quasiparticle across the system changes its state by $\Sigma_{q}^{z}$. This implies that a discrete process of one pair transfer across the system is equivalent to the $\Sigma_{q}^{z}$ transformation. Similarly, a controlled process in which a vortex is moved around a hole results in a discrete $\Sigma_{q}^{x}$ transformation. Moreover, this system allows one to make discrete transformations such as $\sqrt{\Sigma^{x, z}}$. Consider, for instance, a process in which, by changing the total magnetic flux through the system, one half vortex is placed in a center of the system shown in Fig. 1,b and then released. It can escape through the left or through the right boundary, in one case the state does not change, in another it changes by $\Sigma^{x}$. The amplitudes add resulting in the operation $\left(1+i \Sigma^{x}\right) / \sqrt{2}$. Analogously, using the electrostatic gate(s) to pump one charge $2 e$ from one boundary to the island in the center of the system and then releasing it results in a $\left(1+i \Sigma^{z}\right) / \sqrt{2}$ transformation. This type of processes allow a straightforward generalization for the array with many holes: there extra half vortex or charge should be placed at equal distances from the inner and outer boundaries.

The degenerate ground states in the insulating array can be manipulated in the same way as in the superconductor, up to duality (half-vortex $\rightarrow$ Cooper-pair and vice verse). As mentioned above, these states $|0\rangle_{\text {ins }}$ and $|1\rangle_{\text {ins }}$ correspond to the absence or presence of the half-vortex inside the hole. We define by $\tilde{\Sigma}^{x, y, z}$ Pauli operators acting in the space spanned by $|0\rangle_{\text {ins }}$ and $|1\rangle_{\text {ins }}$. The adiabatic change of local magnetic field that drags one half vortex across the system, flips the state of the system, providing us with the implementation of the operator $\tilde{\Sigma}^{x}$ acting on the state of the qubit. Analogously, motion of elementary charge $2 e$ around the hole changes by $\pi$ the relative phase of the states $|0\rangle_{\text {ins }}$ and $|1\rangle_{\text {ins }}$ providing us with the operator $\tilde{\Sigma}^{z}$. Operators $\sqrt{\tilde{\Sigma}^{x, z}}$ can be realized in a way similar to that described for superconductive array. Rotation by an arbitrary angle $U^{x}=\exp \left(i \alpha \tilde{\Sigma}^{x}\right)$ that is an analog of the operator (23) can achieved by modifying (during time $t$ ) parameter $r$ in a way to produce non-negligible amplitude of half-vortex tunnelling $A$ across the system: $\alpha=A t$. In the same way 
two-qubit entanglement operation can be realized, which is an analog of operator (24)):

$$
\tilde{U}_{p, q}^{x}=\exp \left(i t A_{p q} \tilde{\Sigma}_{p}^{x} \tilde{\Sigma}_{q}^{x}\right),
$$

in this case $A_{p q}$ is the half-vortex tunnelling amplitude between holes $p$ and $q$.

\section{Physical properties of small arrays}

\subsection{Superconducting array}

Even without these applications for quantum computation the physical properties of this array are remarkable: it exhibits a long range order in the square of the usual superconducting order parameter: $\left\langle\cos \left(2\left(\varphi_{0}-\varphi_{r}\right)\right)\right\rangle \sim 1$ without the usual order: $\left\langle\cos \left(\varphi_{0}-\varphi_{r}\right)\right\rangle=0$; the charge transferred through the system is quantized in the units of $4 e$. This can be tested in a interference experiment sketched in Fig. $1, b$, as a function of external flux, $\Phi_{m}$ the supercurrent through the loop should be periodic with half the usual period. This simpler array can be also used for a kind of «spin-echo» experiment: applying two consecutive operations $\left(1+i \Sigma_{x}\right) / \sqrt{2}$ described above should give again a unique classical state while applying only one of them should result in a quantum superposition of two states with equal weight.

The echo experiment can be used to measure the decoherence time in this system. Generally one distinguishes processes that flip the classical states and the ones that change their relative phases. In NMR literature the former are referred to as longitudinal relaxation and the latter as transverse one. The transverse relaxation occurs when a vortex is created and then moved around the opening by an external noise. Assuming a thermal noise, we estimate the rate of this process $\tau_{\perp}^{-1} \sim \tilde{t} \exp \left(-E_{V}(L) / T\right)$. Similarly, the transfer of a quasiparticle from the outer to the inner boundary changes the relative phase of the two states, leading to a longitudinal relaxation. This rate is proportinal to the density of quasiparticles, $\tau_{\|}^{-1}=R \exp (-2 r / T)$. The coefficient $R$ depends on the details of the physical system. In an ideal system with some nonzero uniform value of $\epsilon$ (defined above (12)) quasiparticles are delocalized and $R \sim \epsilon / L^{2}$. Random deviations of fluxes $\Phi_{r}$ from half-integer value produce randomness in $\epsilon$, in which case one expects Anderson localization of quasiparticles due to off-diagonal disorder, with localization length of the order of lattice spacing, thus $R \sim \bar{\epsilon} \exp (-c L)$ with $c \sim 1$, and $\bar{\epsilon}$ is the typical value of $\epsilon$. Stray charges induce randomness in the values of $r$, i.e., add some diagonal disorder. When the random part of $r, \delta r$ becomes larger than $\bar{\epsilon}$ the localization becomes stronger:
$R \sim \bar{\epsilon}(\bar{\epsilon} / \overline{\delta r})^{L}$, where $\overline{\delta r}$ is the typical value of $\delta r$. Upon a further increase of stray charge field there appear rare sites where $r_{i}$ is much smaller than an average value. Such site acts as an additional openings in the system. If the density of these sites is significant, the effective length that controls the decoherence becomes the distance between these sites. For typical $E_{V}(L) \approx E_{J} \approx 2 \mathrm{~K}$ the transverse relaxation time reaches seconds for $T \sim 0.1 \mathrm{~K}$ while realistic $\epsilon / r \sim 0.1$ imply that due to a quasiparticle localization in a random case the longitudinal relaxation reaches the same scale for systems of size $L \sim 10$; note that temperature $T$ has to be only somewhat lower than the excitation gap, $2 r$, in order to make the longitudinal rate low.

Most properties of the array are only weakly sensitive to the effect of stray charges: as discussed above, they result in a position-dependent quasiparticle potential energy which has very little effect because these quasiparticles had no kinetic energy and were localized anyway. A direct effect of stray charges on the topologically protected subspace can be also physically described as a effect of the electrostatic potential on the states with even and odd charges at the inner boundary; since the absolute value of the charge fluctuates strongly, this effect is exponentially weak.

\subsection{Insulating array}

The signature of the topological insulator is the persistence of the trapped half flux inside the central hole (see Fig. 4) which can be observed by cycling magnetic field so as to drive the system back and forth between insulating and superconducting states. This trapping is especially striking in the insulator. Experimentally, this can be revealed by driving slowly the array into a superconducting state and then measuring the phase difference between opposite points such as $A$ and $B$ in Fig. 4. In the state with a half vortex the phase difference is $\pi / 2+\pi n$ while it is $\pi n$ in the other state. The $\pi n$ contribution is due to the usual vortices that get trapped in a big hole. This slow transformation can be achieved by changing the strength of weak links using the external magnetic field as a control parameter. The precise nature of the superconductive state is not essential because phase difference $\pi$ between points $A$ and $B$ can be interpreted as due to a full vortex trapped in a hole in a conventional superconductor or due to a $\pi$ periodicity in a topological one which makes no essential difference. These flux trapping experiments are similar to the ones proposed for high- $T_{C}$ cuprates $[18,19]$ with a number of important differences: the trapped flux is half of $\Phi_{0}$, the cycling does not involve temperature (avoiding problems with excitations) and the final state can be either conventional or topological superconductor. 


\section{Conclusion}

We have shown that a Josephson junction array of a special types (shown in Fig. 1 and Fig. 4) have degenerate ground states described by a topological order parameter. The manifold of these states is protected in the sense that local perturbations have exponentially weak effect on their relative phases and transition amplitudes. The simpler array Fig. 1 possesses superconductive state with topological order. Its minor modification shown in Fig. 4 may be brought into both superconductive and insulating «topological» phases in a controllable manner*. Both versions of the array are expected to demonstrate very long coherence time in «spin-echo» - type experiments and to be promising basic elements for scalable quantum computers.

The main building block of the array is the rhombus which has two (almost) degenerate states, in the array discussed here these rhombi are assembled into hexagons but we expect that lattices in which these rhombi form other structures would have similar properties. However, the dynamics of these arrays is described by quartic (or higher) order spin exchange terms which have larger barrier in a quasiclassical regime implying that their parameter $r$ is much smaller than in for the array considered here. This makes them more difficult to built in the interesting regime.

\section{Acknowledgements}

We are grateful to G. Blatter, D. Ivanov, S. Korshunov, A. Larkin, A. Millis, B. Pannetier, E. Serret, and L. Shchur for the discussions and useful comments, and to ENS (Paris), to LPTMS, Orsay, and to LSI, Ecole Polytechnique for their hospitality which allowed this work to be completed.

We acknowledge the support by NSF grant 4-21262. MF was supported by SCOPES program of Switzerland, Dutch Organization for Fundamental research (NWO), RFBR grant 01-02-17759, the program «Quantum Macrophysics» of Russian Academy of Science and the program «Physics of Quantum Computing» of Russian Ministry of Industry, Science and Technology.

1. A. Steane, Rep. Prog. Phys. 61, 117 (1998).

2. A. Ekert and R. Jozsa, Rev. Mod. Phys. 68, 733 (1996).

3. P.W. Shor, in: Proceedings of the 35th Symposium on the Foundations of Computer Science, Los Alamitos, CA:IEEE Press (1994).
4. L. Grover, in: Proceedings of the Annual ACM Symposium on the Theory of Computing, ACM Press, New York (1996).

5. P.W. Shor, Phys. Rev. A52, 2493 (1995).

6. J. Preskill, Proc. Roy. Soc. A454, 385 (1998).

7. A.Yu. Kitaev, Preprint, http://xxx.lanl.gov/abs/quant - ph/9707021 (1997); I. Kant, in: Critique of Pure Reason (1781).

8. X.-G. Wen and Q. Niu, Phys. Rev. B41, 9377 (1990).

9. X.-G. Wen, Phys. Rev. B44, 2664 (1991).

10. M. Mezard, G. Parisi, and M. Virasoro, Spin Glass Theory and Beyond, Word Scientific, Singapore (1997).

11. S.B. Bravyi and A. Kitaev, Preprint, http://xxx. lanl.gov/abs/quant-ph/0003137; M.H. Freedman, A. Kitaev, and Z. Wang, Preprint, http://xxx.lanl.gov/ abs /quant - ph/0001071.

12. L.B. Ioffe, M.V. Feigel'man, A. Ioselevich, D. Ivanov, M. Troyer, and G. Blatter, Nature 415, 503 (2002).

13. L.B. Ioffe and M.V. Feigel'man, Phys. Rev. B66, 224503 (2002)

14. B. Douçot, M.V. Feigel'man, and L.B. Ioffe, Phys. Rev. Lett. 90, 107003 (2003).

15. N. Read and B. Chakraborty, Phys. Rev. B40, 7133 (1989).

16. N. Read and S. Sachdev, Phys. Rev. Lett. 66, 1773 (1991)

17. S. Kivelson, Phys. Rev. B39, 259 (1989).

18. T. Senthil and M.P.A. Fisher, Phys. Rev. Lett. 86, 292 (2001)

19. T. Senthil and M.P.A. Fisher, Phys. Rev. B63, 134521 (2001).

20. L. Balents, S.M. Girvin, and M.P.A. Fisher, Preprint, http:/ / xxx.lanl.gov/abs/cond-mat/0110005.

21. A. Paramekanti, L. Balents, and M.P.A. Fisher, Preprint, http://xxx.lanl.gov/abs/cond-mat/0203171.

22. G. Misguich, D. Serban, and V. Pasquier, Preprint, http: / / xxx.lanl.gov/abs / cond-mat/0204428.

23. O. Motrunich and T. Senthil, Preprint, http://xxx. lanl.gov/abs/cond-mat/0205170.

24. P. Fendley, R. Moessner, and S.L. Sondhi, Preprint, http: / / xxx.lanl.gov / abs / cond-mat/0206159.

25. A.S. Ioselevich, D.A. Ivanov, and M.V. Feigel'man, Preprint, http:/ / xxx.lanl.gov/abs/cond-mat/0206451.

26. G. Blatter, V.B. Geshkenbein, and L.B. Ioffe, Phys. Rev. B63, 174511 (2001).

27. F. Wegner, J. Math. Phys. 12, 2259 (1971).

28. R. Balian, J.M. Droufle, and C. Itzykson, Phys. Rev. D11, 2098 (1975).

29. B. Douçot and J. Vidal, Preprint, http://xxx. lanl.gov/abs/cond-mat/0202115.

30. K. Park and D.A. Huse, Phys. Rev. B64, 134522 (2001).

31. R.F. Bishop, D.J.J. Farrel, and M.L. Ristig, Int. J. Mod. Phys. B14, 1517 (2000).

* It is possible that array shown in Fig. 1 also has topological insulator phase but we cannot prove it. 
32. T. Senthil and M.P.A. Fisher, Phys. Rev. B62, 7850 (2000).

33. N. Read and S. Sachdev, Phys. Rev. Lett. 66, 1773 (1991).

34. L. Balents, M.P.A. Fisher, and S.M. Girvin, Phys. Rev. B65, 224412 (2002).

35. T. Senthil and O. Motrunich, Preprint, http://xxx. lanl.gov/abs/cond-mat/0201320.

36. R. Moessner, S.L. Sondhi, and E. Fradkin, Phys. Rev. B65, 24504 (2002).

\section{Note added in proof}

The approach to the constructing of topologically stable superconductive qubits and anomalous super- conducting states with Cooper-pair paring, that is reviewed in the present article, has been further developed in very recent papers: B. Douçot, M.V. Feigel'man, L.B. Ioffe, and A.S. Ioselevich, Protected Qubits and Chern Simons Theories in JosephsonJunction Arrays, cond-mat/0403712; I.V. Protopopov and M.V. Feigel'man, Theory of 4e Versus 2e Supercurrent in Frustrated Josephson-Junction Rhombi Chain, cond-mat/0405170. 